\section{Armand Trousseau (1801-1867), su historia y los signos de hipocalcemia}

PABLO YOUNG ${ }^{1}$, MARCELO A. BRAVO ${ }^{2}$, MARÍA G. GONZÁLEZ ${ }^{3}$, BÁRBARA C. FINN ${ }^{1}$, MARIANO A. QUEZEL ${ }^{1}$, JULIO E. BRUETMAN ${ }^{1}$

\section{Armand Trousseau (1801-1867), his history and the signs of hypocalcemia}

Armand Trousseau (1801-1867) was born in Tours, France on October 14, 1801. He graduated as a physician in the same city under the direction of Pierre Bretonneau and received his doctorate in 1825 in Paris. He was the first physician to practice tracheotomy in diphtheria and perform thoracenteses to remove air or fluid from the pleural space. He recommended tracheal intubation in different scenarios. He described the first cases of laryngeal tuberculosis and the presence of carpopedal spasm in hypocalcemia, a sign that has his name. He also described the association between migratory thrombophlebitis and neoplasia, which is known as Trousseau's syndrome. Ironically, on January 1, 1867 he diagnosed in himself a deep vein thrombosis of the left upper limb and told one of his disciples "I am lost; I have no doubt about the nature of my disease". He died of gastric cancer at the age of 66 years on June 23, 1867. He carried out an educational and medical work. He instructed his students about the rather instantaneous thought process in clinical medicine. He always conducted his clinical work with the certainty of a sound scientific background. Contemporary physicians should take advantage of the example and lessons of Armand Trousseau.

(Rev Med Chile 2014; 142: 1341-1347)

Key words: History of Medicine; Hypocalcemia; Medical records; Physical examination.

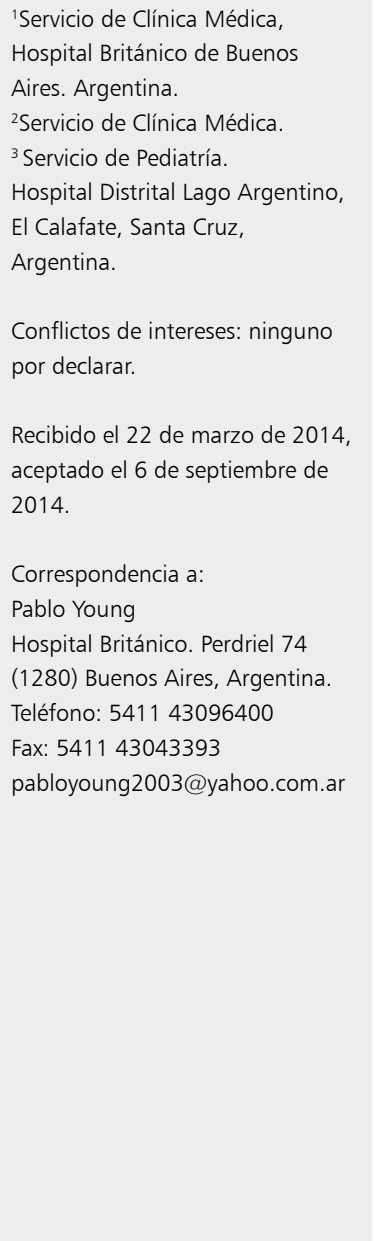

\section{Perspectiva histórica}

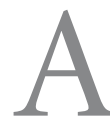

rmand Trousseau (Figura 1) nació en Tours, Francia, el 14 de octubre de $1801^{1-4}$. Estudió en el Colegio de Orleans y en el de Lyon y regresó a su ciudad natal para realizar los estudios secundarios. Estudió medicina en Tours bajo la dirección de Pierre F. Bretonneau (1778-1862) que fue conocido por sus estudios anatomoclínicos de la fiebre tifoidea y la difteria, y en 1825 se doctoró en París con su tesis "Les Lividités cadavériques du canal digestif”. A los 20 años fue profesor de educación secundaria por necesidad ${ }^{3}$.

Fue padre de dos hijos, una mujer que lo acompañó en sus últimos momentos y Georges Phillipe Trousseau (1833-1894) que fue médico en Hawaii.
Su nieto fue un distinguido oftalmólogo: Armand Henri Trousseau (1856-1910)

Ingresó como alumno interno en la Maison Royale de Charenton, hoy Hôpital Esquirol, donde profundizó el estudio de las enfermedades mentales bajo la tutela de Jean-Étienne Dominique Esquirol (1772-1840) y, aprovechando la proximidad de la Escuela Nacional de Veterinaria de Alfort, comenzó sus investigaciones de anatomía y patología comparadas ${ }^{1}$. Estuvo en el Hôpital Necker y en el Hospital Infanta de Jesús donde estudió y enseñó enfermedades pediátricas. En 1827 se incorporó a la Facultad de Medicina de París con el trabajo "An membranae mucosae gastro-intestinalis inflammatio, certis signis, tum in vivo, tum in cadavere dignoscitur". El mismo día que fue elegido Profesor de Medicina en la Facul- 


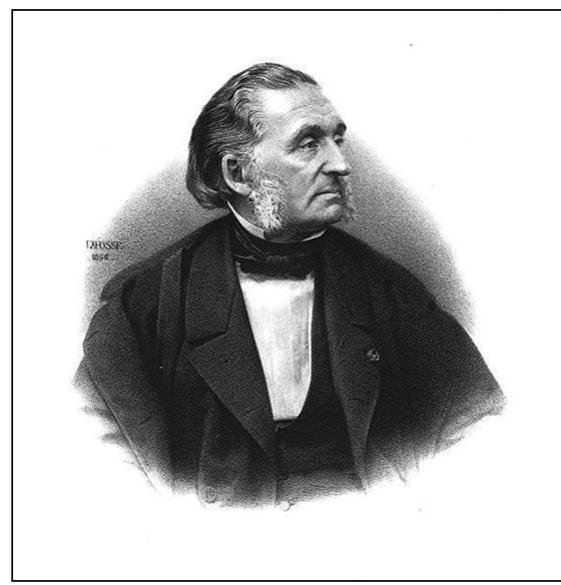

Figura 1. Dr. Armand Trousseau. En: http://es.wikipedia.org/ wiki/Armand_Trousseau; consultado 4/3/2014.

tad de París, fue nombrado Jefe de Cirugía en el Hôspital de Tours y por sus cualidades diagnosticó y operó un absceso perirrenal idiopático o flemón perinefrítico idiopático de Trousseau o sea no debido a infección renal ascendente (Tabla 1) ${ }^{1-4}$.
En 1828 el gobierno le encargó el estudio de las epidemias y endemias de la región del Mediodía francés (sur de Francia) ${ }^{1}$. También formó parte de la comisión que estudió la fiebre amarilla en Gibraltar, junto a Pierre C. A. Louis (1787-1872). Publicaron en 1830 "Documents recueillis par la commision française envoyée à Gibraltar pour observer la fièvre jaune qui a régné dans cette place”. En 1831 sustituyó por concurso a Joseph Récamier (1774-1852) como médico del Hôtel-Dieu². En 1834 fundó junto a Henri Gouraud (1807-1874) y Jacques Lebaudy Trousseau el "Journal connaissances médico-chirurgicales" y en ese año utilizó el efecto placebo mencionando que era tan o más efectivo que las diluciones homeopáticas ${ }^{6}$. La Academia de Medicina lo premió en 1837 por su trabajo "Traité practique de la phthisie laryngée, de la laryngite chronique et des maladies de la voix".

En el año 1839 pasó al Hôpital Saint-Antoine y ganó el concurso de la cátedra de terapéutica y farmacología de la Facultad de Medicina para sustituir a Jean L. Alibert (1768-1837) fundador de la Escuela Francesa de Dermatología ${ }^{2,4}$. A partir de 1850 sustituyó a Auguste Chomel (1788-1858)

\section{Tabla 1. Descripciones de Armand Trousseau}

\begin{tabular}{|l|}
\hline Descripción de enfermedad, entidad, síndrome, signo o procedimiento \\
Enfermedad de Trousseau o tuberculosis ganglionar \\
Tuberculosis laríngea \\
Pseudomembranas en la difteria \\
Realizó la primera toracocentesis \\
Enfermedad de Trousseau o síndrome de Gilles de la Tourette \\
Utilizó el término "afasia" \\
Neuralgia epileptiforme de Trousseau o neuralgia del trigémino \\
Puntos de Trousseau o puntos dolorosos por la compresión de las vértebras \\
Raya roja meningítica de Trousseau como signo de dermografismo en la meningitis \\
Cuerpos de Trousseau-Lallemand o proteinuria de Bence Jones \\
Flemón perinefrítico idiopático de Trousseau o absceso perirrenal idiopático \\
Dio el nombre de enfermedad de Addison a la insuficiencia suprarrenal primaria \\
Signo de Trousseau o mano de partero en la hipocalcemia \\
Prueba Trousseau-von Bonsdorff \\
Signo de Trousseau o impotencia funcional debido al dolor en trombosis venosa \\
Síndrome de Trousseau o asociación de tromboflebitis migratoria con cáncer visceral \\
Hemocromatosis o enfermedad de Trousseau \\
Signo del Bronce de Trousseau en neumotórax \\
Vértigo gástrico o vértigo de Trousseau \\
\hline
\end{tabular}




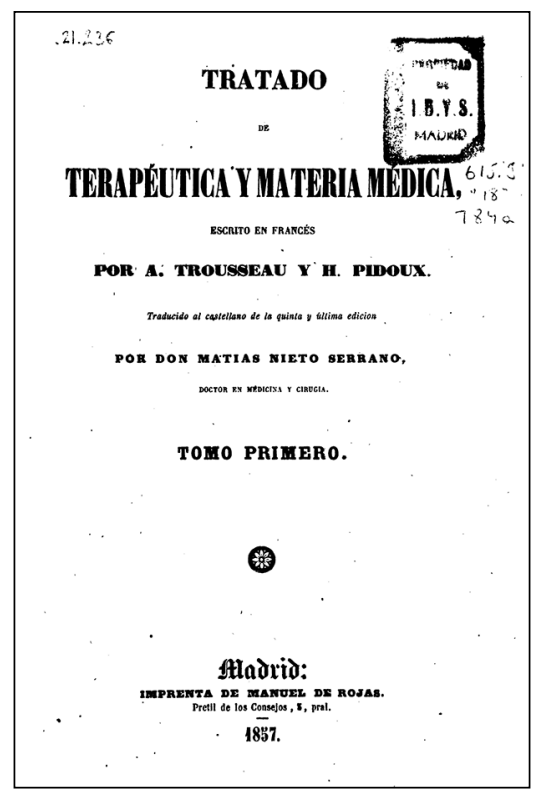

Figura 2. A. Tratado de Terapéutica y Materia Médica y B. Clínicas Médicas del Hotel Diu. https:// www.google.com.ar/search? $q=a r$ mand + trousseau \&source $=\operatorname{lnms} \&$ $\mathrm{tb} \mathrm{m}=\mathrm{isch} \& \mathrm{sa}=\mathrm{X} \& \mathrm{e} i=67 \mathrm{bvU} 4 \mathrm{fpC}$ If7oAT 3IHIDg\&sqi $=2 \&$ ved $=0 C A$ YQ_AŪoAQ\&biw $=1093 \& b i h=490$; consultado 16/8/2014. como profesor de clínica médica del Hôtel-Dieu². En 1864 retomó la cátedra de terapéutica y materia médica en la Charité. Sus contribuciones más significativas se deben al razonamiento clínico fundado en la analogía, la inducción, deducción, diagnóstico diferencial y su sustento anatomopatológico, lo que se observa en sus dos grandes obras, el "Traité de thérapeutique et de matière médicale" que publicó junto con H. Pidoux entre 1836 y 1839 y las "Clinique médicale de l'HôtelDieu" de París que aparecieron entre 1861 y 1862 (Figura 2) $^{1-4,8,9}$.

Entre sus discípulos más reconocidos se encuentran Charles E. Lasègue (1816-1883) recordado por el signo que lleva su nombre; Georges P. Dieulafoy (1839-1919) uno de los grandes clínicos franceses y recordado por la lesión submucosa gástrica que lleva su nombre; Guillaume B. A. Duchenne (1806-1875) quien describió la distrofia muscular y fue uno de los fundadores de la neurología moderna; Maurice Krishaber (1836-1883) médico húngaro afincado en Francia quien describió los cuadros de despersonalización; y Jacob M. Da Costa (1833-1900) quien estudió las enfermedades funcionales del corazón y en 1871, describió en soldados un síndrome caracterizado por un corazón irritable o lábil, conocido luego como síndrome de Da Costa o "astenia neurocirculatoria" o "corazón de soldado"1-4.
Fue elegido miembro de la Academia de Medicina en 1856 y recibió la Legión de Honor en 1858. A partir de 1866 fue Profesor Honorario. El 1 de enero de 1867 se autodiagnosticó una trombosis venosa profunda (flegmasia alba dolens) de una de sus extremidades y le comentó al Dr. Peter, discípulo suyo: "estoy perdido, no tengo dudas sobre la naturaleza de mi enfermedad", ya que poco tiempo antes había descripto la asociación entre cáncer y trombosis. Murió de un cáncer gástrico a la edad de 66 años, el 23 de junio de $1867^{4}$. Dijo Dieulafoy: "tuvo lenta agonía que cursó con serenidad y estoicidad, y en ese tiempo las discusiones médicas fueron su pasatiempo favorito"4. El funeral se efectuó en la Iglesia de la Madeleine y fue enterrado en el cementerio de Père-Lachaise, en París ${ }^{1,2}$.

El viejo hospital Faubourg Saint-Antoine, que databa de 1674, se llamó en 1880 hospital Trousseau en homenaje a él (Figura 3). En 1895, el hospital Trousseau fue reemplazado por tres hospitales: el Bretonneau, el Herold y el nuevo Trousseau.

\section{Contexto político y científico en el siglo XIX}

La edad contemporánea abarca un período que va desde la Revolución Francesa (1789-1799) hasta la actualidad. En el siglo XIX la medicina se 


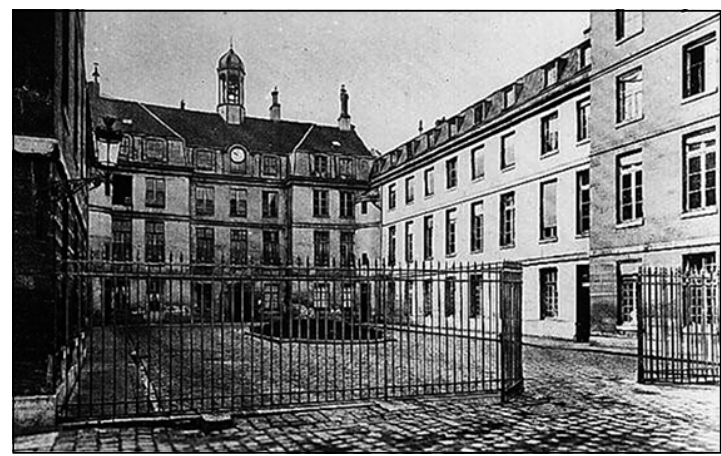

Figura 3. Viejo hospital Trousseau. En: https://encryptedtbn0.gstatic.com/images? q =tbn:ANd9GcSwLaHUVnKXRsJcb dkaRtP8TE0V6a-aQObT1X5kQOf3Wwbn0Ty87Q; consultado $4 / 3 / 2014$

relaciona directamente con los fenómenos migratorios, los hacinamientos en las ciudades y las precarias condiciones de vida de la clase trabajadora propios de la Revolución Industrial (1776 a 1830, aproximadamente). Su consecuencia fue la proliferación de enfermedades infecciosas (sífilis, tuberculosis) o relacionadas con la mala alimentación (pelagra, raquitismo, escorbuto). Estos movimientos tuvieron una clara influencia sobre la sociedad y sobre la ciencia y la medicina. En lo que se refiere a la clínica, la Primera Escuela Francesa surgió en París en plena Revolución y Trousseau fue una de sus más encumbradas figuras ${ }^{10}$.

Dichos movimientos son cruciales para entender el origen de la medicina social de Rudolf Virchow (1821-1902) y el sistema de salud pública de Edwin Chadwick (1800-1890) que darían lugar a la actual medicina preventiva. La Revolución Industrial, con el agregado de las numerosas guerras y revoluciones, generarían un desarrollo científico generalizado que contribuiría a la instauración de condiciones técnicas para el triunfo de la asepsia, la anestesia y la cirugía. En 1848 comenzó a emplearse el éter para sedar a los pacientes antes de la cirugía y a finales de ese siglo Luis Pasteur, Robert Koch y Joseph Lister demostrarán inequívocamente la naturaleza etiológica de los procesos infecciosos mediante la teoría microbiana ${ }^{11}$.

El movimiento ideológico que dio sustento a esta etapa es el positivismo, sistema filosófico que postula que todos los conocimientos deben basarse en datos de la experiencia, de la ciencia, excluyendo todo intento de investigación metafísica. El positivismo nació con las ideas del filósofo francés
Augusto Comte (1798-1857), quien sostenía que el pensamiento humano había pasado por una etapa teológica-religiosa, luego otra metafísica y finalmente llegado a la positiva o científica. En este período ejerció asimismo una clara influencia otro filósofo, Étienne B. Condillac (1714-1780), creador del "sensualismo", teoría del conocimiento según la cual el saber humano se reduce a las ideas concretas de lo que se es capaz de captar a través de las sensaciones. Por ejemplo, en el caso de la auscultación, René Théophile Hyacinthe Laënnec (1781-1826) hizo uso del sensualismo auditivo ${ }^{10,11}$.

Este siglo verá nacer la teoría de la evolución de las especies, de Charles Darwin (1809-1882), expresión antropológica del positivismo científico. La realidad puede medirse, comprenderse y predecirse mediante leyes, que a su vez van siendo corroboradas por los sucesivos experimentos. Por ese camino avanzan la astronomía (Laplace, Foucault), la física (Poincaré, Lorentz), la química (Dalton, Gay-Lussac, Mendeleiev) y la genética (Gregor Mendel, 1822-1884) ${ }^{11}$.

La figura médica por excelencia de este siglo fue el alemán Rudolf Virchow quien postuló la teoría de "toda célula proviene de otra célula" y explicó a los organismos vivos como estructuras formadas por células. Además lo une a Trousseau su interés por la trombosis y la descripción de su triada fisiopatológica.

En este siglo compiten y se complementan dos concepciones médicas capitales: el criterio anátomo-clínico, encumbrado por la obra de Laënnec y el criterio fisiopatológico que recibe el vigoroso impulso de Claude Bernard (1813-1878) y, con él, la prédica del razonamiento experimental, base de la investigación biológica.

\section{Sus descripciones}

Dejó una huella brillante en el estudio clínico de la tuberculosis laríngea (realizó la primera descripción completa), por lo que se le otorgó el premio de la Academia de Medicina en 1837 (Tabla 1) 1-4,12.

Clasificó las anginas por las pseudomembranas que describió y la difteria en tóxicas o no tóxicas. Mencionó que la parálisis diftérica se debía a un "veneno" que libera el germen, corroborado años después por Pierre P. E. Roux (1853-1933) y Alexandre Yersin (1863-1943) al identificar la to- 
xina. Uno de sus aforismos decía que la escarlatina no ama la laringe, como si lo hace la difteria ${ }^{1,3,4}$.

Popularizó el uso de la traqueostomía, término implementado por Lorenz Heister (1683-1758) en 1718 , como tratamiento del croup diftérico (en papiros egipcios del 3.600 a.C. ya figura esta técnica). Fue el primero en drenar la cavidad pleural (toracocentesis, del griego thōrāko- "tórax" y kentēsis- "perforación”) en 1833, y promulgó la intubación orotraqueal ${ }^{12}$.

Como Laënnec, Trousseau estudió y describió de manera magistral el asma (a la cual llamó la epilepsia del pulmón), dado que pensó que era una enfermedad nerviosa retornando a la antigua teoría de Avicena (980-1037) reproducida por William Cullen (1710-1790) y Bretonneau, y la trató con broncodilatadores inhalados derivados de plantas. Recomendó lo que posteriormente se llamó "cigarro medicinal de Trousseau" quemando hojas secas de "Datura" que contienen estramonio y su alcaloide la l-hiosciamina y dentro de los broncodilatadores orales utilizó la belladona y atropina ${ }^{13}$.

En el tratamiento del raquitismo utilizó el aceite de hígado de bacalao ${ }^{14}$. Escribió sobre osteomalacia, corea, malaria, enfermedad de Parkinson, migraña, gota, y hemorroides ${ }^{1-4,15-17}$. Describió el vértigo gástrico o vértigo de Trousseau cuyo gatillo está en el estómago y fue el primero en utilizar la palabra "afasia"16.

Se llama síndrome de Trousseau a la tromboflebitis migratoria (trombosis venosa superficial) de origen neoplásico, habitualmente por cáncer visceral (comúnmente de estómago y páncreas). Si bien describió la trombosis venosa superficial, hoy el término se utiliza indistintamente para las trombosis superficiales o profundas asociadas a malignidad $^{1-4,18-20}$. Trousseau reconoció que no fue el primero en registrar esta asociación pues un discípulo suyo, J. Werner, reportó el caso de un hombre de 46 años y Rudolf Virchow informó un caso de cáncer de estómago y trombosis en ambos miembros inferiores y edema doloroso en miembro superior izquierdo ${ }^{2}$. En este contexto se conoce como signo de Trousseau a la impotencia funcional debida al dolor en las trombosis venosas profundas.

Varias enfermedades o síndromes llevan su nombre, como la linfademia aleucémica o tuberculosis ganglionar ${ }^{1-4}$. El dermografismo en la piel de un paciente con meningitis se conoce como mancha o raya roja meningítica de Trousseau ${ }^{1}$. Jonathan Hutchinson (1828-1913) y Armand Trousseau (1801-1867) confirman la insuficiencia suprarrenal primaria y este último la denomina enfermedad de Addison. Además, popularizó los epónimos de linfoma de Hodgkin y enfermedad de Graves ${ }^{21,22}$. La neuralgia del trigémino se conoce como neuralgia epileptiforme de Trousseau, si bien había sido descripta en el año 1773 por John Fothergill (1712-1780) y en el siglo II d.C. por Areteo de Capadocia. Se conoce como "puntos de Trousseau" a los puntos dolorosos por la compresión de las vértebras dorsales y lumbares en ciertos casos de neuralgias ${ }^{1}$.

La hemocromatosis fue descubierta por Armand Trousseau en 1865, quien describió un síndrome clínico representado por diabetes, hiperpigmentación cutánea y cirrosis hepática. A esta asociación, en 1871 Charles Emile Troisier (18441919) la llamó diabetes bronceada. La hemocromatosis, o enfermedad de Trousseau, también se conoce como enfermedad de Troisier-HanotChauffard, síndrome de Leschke, o síndrome de Von Recklinghausen-Appelbaum. Utilizó preparaciones en base de plantas para el tratamiento de la anemia y de quinina para la malaria ${ }^{1,4}$.

Después de su muerte, en 1873, fueron publicados algunos de sus trabajos en tres volúmenes de las “Clinique Médicale de L'Hôtel Dieu de Paris" y sus observaciones de lo que se conocería luego como síndrome Gilles de la Tourette. Georges Gilles de la Tourette (1857-1904) re-describe el cuadro en 1885 y en su artículo original menciona brevemente el trabajo de Trousseau y dice que éste presentó una pequeña serie que no amerita un análisis en profundidad ${ }^{23}$.

Por último, se conoce como síndrome de ParisTrousseau o de Jacobsen a un trastorno hereditario caracterizado por la tendencia hemorrágica leve asociada con la supresión del cromosoma 11q y que lleva este nombre por haber sido descripto en el Hospital Trousseau y no por Armand Trousseau ${ }^{24}$.

\section{Hipocalcemia, sus síntomas y signos}

La hipocalcemia se manifiesta clínicamente por irritabilidad neuromuscular marcada y progresiva que comienza con calambres (cuando son faciales se llama facie de Uffenheimer), parestesias periorales, entumecimiento digital y se continúa 
con tetania, laringoespasmo y convulsiones. Se ha observado además broncoespasmo, ansiedaddepresión-trastornos cognitivos, edema de papila, paro respiratorio y a nivel electrocardiográfico prolongación del intervalo QT y arritmias ventriculares ${ }^{25}$.

Se han reportado como hallazgos alteraciones ectodérmicas (dermatitis, eccema, psoriasis, alopecia, surcos transversales en las uñas), candidiasis en el hipoparatiroidismo y cataratas en la hipocalcemia crónica.

Así como la mano de partero o signo de Trousseau es manifestación cardinal de la enfermedad, se pueden encontrar ciertos hallazgos en el examen físico en algunos de estos pacientes, por orden alfabético:

Signo de Chvostek: Contracción de músculos faciales al estimular la zona del nervio facial anterior (signo de tetania latente).

Signo de Erb: Umbral bajo frente a estímulos eléctricos que provoca espasmo carpo pedal (símil Trousseau).

Signo de Escherich: Un golpe en la piel de la comisura labial causa protrusión de los labios.

Signo de Frankl Hochwart: Al realizar la maniobra para Trousseau, la respuesta se observa en la mano contralateral.

Signo de Hoffmann: Se provoca flexión de la falange distal del tercer dedo, puede aparecer flexión de la última falange de los dedos índice y pulgar (hiperexitabilidad neuromuscular por lo cual puede ser también signo piramidal).

Signo de Hoffmann: La irritación mecánica del trigémino en sus puntos de emergencia provoca dolor local e inmediato (sensitivo).

Signo de Kashida: Espasmo de los músculos e hiperestesia producidos por la aplicación de calor o frío.

Signo de Khan y Falta: Al realizar la maniobra para Trousseau puede agregarse un espasmo vascular con la consiguiente palidez de los dedos.

Signo de Lust: Flexión dorsal y abducción del pie al percutir el peroneo, justamente por debajo de la cabeza del peroné mientras la rodilla se encuentra relajada en ligera flexión.

Signo de Pool (del brazo): Contracción de los músculos del brazo después del estiramiento del plexo braquial elevando el brazo sobre la cabeza con el antebrazo extendido.

Signo Schlesinger o fenómeno de Pool: Espasmo de los músculos extensores de la rodilla y de los músculos de la pantorrilla cuando la pierna extendida se flexiona en la cadera.

Signo de Schultze: Al percutir la lengua ésta se deprime y muestra un dorso cóncavo.

Signo de Trousseau: Espasmo carpopedal al inflar manguito de presión por encima de la sistólica por 3 min (signo de tetania latente).

Signo de Weiss: Un golpe en la piel periorbicular causa cierre de los párpados.

La respuesta espasmódica de músculos faciales, ante la estimulación mecánica del séptimo par craneano, fue descrita por el cirujano austriaco de origen checo Frantisek Chvostek (1835-1884) en $1876^{23}$.

El signo de Chvostek tiene tres variables en su modalidad de exploración: 1) La estimulación mecánica por frote del lóbulo de la oreja provoca la contractura del músculo orbicular de los párpados, los elevadores del ala de la nariz y los músculos de la comisura labial; 2) La percusión de un punto situado un centímetro por debajo del proceso cigomático del hueso temporal y dos centímetros delante del lóbulo de la oreja, genera una respuesta similar a la anterior aunque ausente en los músculos orbiculares; y 3) Al percutir sobre los dos tercios superiores de la línea que une la comisura labial con el arco cigomático, se contrae la comisura labial. Algunos autores opinan que esta respuesta puede considerarse un reflejo. Estos se conocen como Chvostek I, II o III respectivamente ${ }^{25,26}$.

Este signo puede resultar positivo en algunas enfermedades que cursan sin hipocalcemia, como mixedema, escarlatina, difteria y sarampión. El signo de Chvostek puede ser positivo hasta en $25 \%$ de las personas sanas y negativo hasta en 29\% de pacientes con hipocalcemia. Por lo anterior se considera al signo de Chvostek como un indicador grueso de irritabilidad neuromuscular y no de hipocalcemia solamente.

El signo de Trousseau es el signo de hipocalcemia por antonomasia: cuando ejercemos una presión sobre un tronco nervioso (el cubital, por ejemplo) o una compresión mecánica de la arteria braquial (insuflación del manguito del esfingomanómetro $20 \mathrm{mmHg}$ por encima de la presión sistólica por $3 \mathrm{~min}$ ), se generan en toda la extremidad cambios isquémicos incrementando la sensibilidad de los músculos a la hipocalcemia, provocando espasmo carpopedal: flexión de la muñeca y de las articulaciones metacarpofalán- 
gicas, hiperextensión de los dedos y la flexión del dedo pulgar sobre la palma de la mano ${ }^{1,3,7}$. La mano adopta una forma cónica llamada "mano de partero o comadrona”. En adición al hallazgo visual, el paciente refiere parestesias en los dedos, fasciculaciones, sensación incontrolable de que "los dedos se van a torcer" y calambres. Este signo es más sensible y específico que el de Chvostek para la tetania hipocalcémica. Se observa también en hipomagnesemia. Algunos proponen que una vez desinflado el brazalete, se pida al paciente que hiperventile a razón de 40 veces por minuto. Esta prueba llamada de Trousseau-von Bonsdorff debe mantener el espasmo carpopedal y se usa como confirmatoria. El 94\% de los pacientes con hipocalcemia comprobada presentó una prueba positiva, y esto se puede observar hasta en $1 \%$ de pacientes sanos ${ }^{25}$.

Los médicos del nuevo milenio deberíamos reconocer en Armand Trousseau un ejemplo a ser continuado.

\section{Referencias}

1. Peumery JJ. [Armand Trousseau (1801-1867), French physician par excellence]. Hist Sci Med 2003; 37: 151-6.

2. Khorana AA. Malignancy, thrombosis and Trousseau: the case for an eponym. J Thromb Haemost 2003; 1: 2463-5.

3. Bariéty M. [Tribute to Armand Trousseau (14 October 1801-23 June 1867)]. Bull Acad Natl Med 1967; 151: 627-35.

4. Dieulafoy G. Lecon D’ouverture. Clinique Médicale de L’Hotel Dieu de París, 1896-1897. París año 1898 p 1-22.

5. Peumery JJ. [The adventuresome existence of Georges Phillippe Trousseau (1833-1894)]. Hist Sci Med 2005; 39: 197-202.

6. Reubi FC. [Armand Trousseau (1801-1867) and the placebo effect]. Schweiz Med Wochenschr 1986; 116: 27-8.

7. Trousseau A, Belloc H. Traité pratique de la phtisie laryngée, de la laryngite chronique, et des maladies de la voix. París, Francia: Editorial J-B Baillière; 1837. p 488-97.

8. Trousseau A, Pidoux H. Traité de thérapeutique et de maitère médicale. 3 vols. París, Francia. Editorial Béchet; 1836-39. Disponible en: http://gallica.bnf.fr/ark:/12148/ bpt6k77324x.image.r=trousseau.f5.langFR [Consultado el 16 de agosto de 2014].

9. Trousseau A. Clinique médicale de l'Hôtel-Dieu de Paris,
Tome 1. París, Francia: Editorial JB. Baillière; 1868. p 563-697.

10. Marongiu FA. La clínica médica en la edad contemporánea. En: Historia de la clínica médica y de la medicina interna. Buenos Aires, Argentina: Editorial Edimed; $1^{\circ}$ Ed; 2012. p. 65-165.

11. Laín Entralgo P. Evolucionismo, positivismo, eclectismo (Siglo XIX). En: Historia de la Medicina. Barcelona, España: Editorial Salvat; 1 Ed; 1978. p. 387-415.

12. Legent F. [Armand Trousseau, laryngologist]. Hist Sci Med 2007; 41: 83-94.

13. Cohen SG. Asthma among the famous. Armand Trousseau (1801-1867), French physician. Allergy Asthma Proc 1997; 18: 40-2.

14. Dunn PM. Professor Armand Trousseau (1801-67) and the treatment of rickets. Arch Dis Child Fetal Neonatal 1999; 80: 155-7.

15. Trousseau A. Des diverses especes de chorées. Clinique Médicale de L’Hotel Dieu de París 1873; 2: 264-71.

16. Lebrun Y. Portraits in aphasia: Armand Trousseau (18011867). Eur J Disord Commun 1993; 28: 103-8.

17. Pearce JM. Armand Trousseau-some of his contributions to neurology. J Hist Neurosci 2002; 11: 125-35.

18. Anning ST. The historical aspects of venous thrombosis. Med Hist 1957; 1: 28-37.

19. Donati MB, Lorenzet R. Thrombosis and cancer: 40 years of research. Thromb Res 2012; 129: 348-52. Review.

20. Trousseau A. Phlegmasia alba dolens. Clinique médicale de l'Hôtel-Dieu de París 1865; 654-712.

21. Young P, Finn BC, Pellegrini D, Bruetman JE. Hutchinson (1828-1913), su historia, su tríada y otras tríadas de la medicina. Rev Med Chile 2010; 138: 383-7.

22. Young P, Finn BC, Bruetman JE. La enfermedad de graves, signos y síntomas. An Med Interna (Madrid) 2007; 24: 505-8.

23. Rickards H, Woolf I, Cavanna AE. "Trousseau's disease:" a description of the Gilles de la Tourette syndrome 12 years before 1885. Mov Disord 2010; 25: 2285-9.

24. Favier R, Jondeau K, Boutard P, Grossfeld P, Reinert P, Jones $\mathrm{C}$, et al. París-Trousseau syndrome: clinical, hematological, molecular data of ten new cases. Thromb Haemost 2003; 90: 893-7.

25. Rosenberger K. Management of electrolyte abnormalities: hypocalcemia, hypomagnesemia, and hypokalemia. J Am Acad Nurse Pract 1998; 10: 209-17. Review.

26. Zadina R. Dr. Frantisek Chvostek. 11 March 1835-6 November 1884. Cas Lek Cesk 1984; 123: 1608-9. 